\title{
Editorial: Proceedings of the International School on Magnetic Resonance and Brain Function - XII Workshop
}

\author{
Federico Giove ${ }^{1,2 *}$ and Itamar Ronen ${ }^{3}$ \\ ${ }^{1}$ MARBILab, Centro Fermi - Museo Storico Della Fisica e Centro di Studi e Ricerche Enrico Fermi, Rome, Italy, ${ }^{2}$ Fondazione \\ Santa Lucia IRCCS, Rome, Italy, ${ }^{3}$ C. J. Center for High Field MRI Research, Leiden University Medical Center, \\ Leiden, Netherlands
}

Keywords: International School on Magnetic Resonance and Brain Function (ISMRBF), MRI and fMRI, MRI techniques, multimodal MRI, neuroscience

\section{Editorial on the Research Topic}

Proceedings of the International School on Magnetic Resonance and Brain Function - XII Workshop

This Special Research Topic includes the proceedings of the XII workshop of the International School on Magnetic Resonance and Brain Function (ISMRBF), held between April 30th and May, 6th 2016 in Erice (Italy) at the premises of the Ettore Majorana Foundation and Centre for Scientific Culture. ISMRBF is a biennial event that gathers scientists whose research is focused on the crucial intersection between technological advances in MRI and emerging applications in neuroscience.

Indeed, many recent improvements in MRI instrumentation and techniques have been triggered

OPEN ACCESS

Edited and reviewed by: Ewald Moser

Medizinische Universität Wien, Austria

*Correspondence:

Federico Giove

federico.giove@uniroma1.it

Specialty section: This article was submitted to Biomedical Physics, a section of the journal

Frontiers in Physics

Received: 07 February 2018 Accepted: 12 February 2018

Published: 06 March 2018

Citation:

Giove F and Ronen I (2018) Editorial:

Proceedings of the International

School on Magnetic Resonance and

Brain Function - XII Workshop.

Front. Phys. 6:18.

doi: 10.3389/fphy.2018.00018 by the increasing demands from a vast community of neuroscientists, which are exploiting MRI as one the most powerful tools for non-invasive investigation of human brain structure and function. Another long lasting topic covered by ISMRBF is the use of multimodal and multiparametric approaches. MRI is intrinsically multiparametric, thanks to the flexibility of the framework for contrast generation, that can be made sensitive to multiple biophysical phenomena. MRI can be also combined with other distinct modalities, such as electrophysiology, optical imaging and PET based molecular imaging. Fusion of information within- and across modalities has shown an excellent potential to complement the strengths and weaknesses of MRI as a stand-alone technique.

The papers included in this Research Topic offer a broad overview of the subjects covered by the Workshop. Mangia et al. contributed a paper that shows the potential of multiparametric MRI in application to neurological diseases, characterizing how Parkinson's disease and idiopathic rapid eye movement sleep behavior disorder are associated with tissue properties in subcortical regions. The authors exploited a battery of conventional (diffusion tensor imaging, DTI) and non-conventional metrics (adiabatic and non-adiabatic rotating frame relaxation), and concluded that rotating frame relaxation combined with functional connectivity may provide sensitive and specific signatures of progression of neurological diseases. The paper by Lehto et al. used similar MRI approaches on rats. In this case, MRI outcomes were correlated with myelin content in pharmacologically induced demyelinating lesions. Techniques based on non-adiabatic rotating frame relaxation showed good specificity and excellent sensitivity in the detection of lesions, and good quantitative correlation with actual myelin content. In comparison, the sensitivity reached by a battery of more conventional MRI approaches was lower. Authors concluded that non-adiabatic rotating frame relaxation is a valuable technique for the investigation of demyelinating diseases. 
The contribution by Havlicek et al. expanded the multimodal approach to functional MRI. By combining multiple MRI and electrophysiological datasets from humans, non-human primates and cats, they discussed the generative model for BOLD (BloodOxygenation Level-Dependent) they recently introduced, and showed that it can explain the dynamic relationship between haemodynamic and neural events underlying the generation of the BOLD contrast. Hawsawi et al. dealt with multimodal combination of MRI and electrophysiological measurements in humans from the complementary point of view of patient safety. In their paper, the authors explored the safety issues related to simultaneous MRI and scalp or intracranial EEG, by identifying the physical phenomena that play a role in patient safety and reviewing the relevant literature. The authors concluded that risks can be managed, but that simultaneous invasive electrophysiology and MRI is characterized by a set of potential health hazards so complex, that careful case by case evaluation is needed.

The paper by Bednarik et al. is a retrospective study on patients affected by type 1 diabetes, investigating the connection between the pathology itself, impaired hypoglycaemia awareness, and cortical atrophy. Authors showed that type 1 diabetes is associated with gray matter atrophy compared to matched controls. The effect was larger in subjects with impaired hypoglycaemia awareness, likely because of increased exposure to hypoglycaemic episodes. Authors indicated that future studies should clarify whether the reported subtle reductions of gray matter volume are associated with cognitive impairment. The review by Van Schependom and Nagels discusses the relationship between neuroimaging and cognitive scores in multiple sclerosis (MS). The authors highlighted that improving the capability of neuroimaging-derived metric to assess pathology-related features can be useful not only for the characterization of the pathophysiological mechanisms underlying MS damage, but also to improve and speed-up the development of innovative therapies.

Tommasin et al. studied how the performance of a sustained cognitive stimulus affects the features of slow BOLD oscillations underlying functional connectivity within the Default Mode Network (DMN). They found that the stimulus reduced both amplitude of oscillation and functional connectivity within the DMN, but that the coupling between the reported changes was spatially heterogeneous and likely frequency specific. They concluded that the coupling between amplitude of BOLD fluctuations and functional connectivity is a potentially useful parameter to study brain networks. Bordier et al. presented a simulation work aimed at exploring optimal procedures for sparsification in graph theory analysis of topology of functional networks. Sparsification is often needed to simplify the processing and the interpretation of networks, however it is prone to over-pruning the network, and the identification of an optimal threshold is an open issue. The authors used synthetic data to show that percolation data-driven approaches can be used to identify a threshold that maximize the information of the pruned network.

The next couple of papers deal with MR approaches to study neurophysiology and neurochemistry. Sonnay et al. reviewed the applications of ${ }^{13} \mathrm{C}$-MR to brain energetics, and in particular how the combination of advanced MR techniques, mathematical models of metabolism and nutrients transport, and increasing magnetic fields can help the understanding the specific metabolic response to stimulation within astrocytes and neurons, as well as the metabolic interactions between these cells. Driver et al. studied an important issue regarding the measurement of brain oxygen consumption $\left(\mathrm{CMR}_{\mathrm{O}_{2}}\right)$ by $\mathrm{MR}$ via the socalled calibrated-BOLD method, i.e., the usual isometabolic assumption during hypercapnia. The authors showed that their new approach, using the most relaxed and physiologically sensible hypothesis of linear dependence between a graded hypercapnic challenge and $\mathrm{CMR}_{\mathrm{O}_{2}}$, allows more accurate estimates of basal $\mathrm{CMR}_{\mathrm{O}_{2}}$ as well as activation-related $\mathrm{CMR}_{\mathrm{O}_{2}}$ change.

Finally, Nam and Kim tackled the issue of decoding brain states by fMRI, by showing that brain reading can be achieved non-invasively even in the motor domain. Specifically, they demonstrated that directional arm movement can be predicted in humans exploiting simple linear encoding models.

The next workshop of the ISMRBF will be held in Erice, April 22-29 2018. The focus will be, as usual, on brain functional and microstructural MRI and compatible approaches. Information about the workshop can be found at the website http://ismrbf. marbilab.eu.

\section{AUTHOR CONTRIBUTIONS}

All authors listed have made an equal contribution to the work, and approved it for publication.

Conflict of Interest Statement: The authors declare that the research was conducted in the absence of any commercial or financial relationships that could be construed as a potential conflict of interest.

Copyright (c) 2018 Giove and Ronen. This is an open-access article distributed under the terms of the Creative Commons Attribution License (CC BY). The use, distribution or reproduction in other forums is permitted, provided the original author(s) and the copyright owner are credited and that the original publication in this journal is cited, in accordance with accepted academic practice. No use, distribution or reproduction is permitted which does not comply with these terms. 